\title{
Reform der Justizstrukturen - technokratisch-fiskalisch oder substantiell?
}

Hans-Ernst Böttcher

ch erinnere mich an eine Fortbildungstagung der französischen Richterhochschule Ecole Nationale de la Magistrature (ENM) im Herbst 2001 in Paris: Wie so häufig in den letzten Jahren bei justizbezogenen Fortbildungsveranstaltungen in ganz Europa, ging es um die Reform der Strukturen der Justiz, in diesem Fall um die internen Verwaltungsstrukturen der Gerichte. Die ten Organs der Richterschaft (des Präsidiums) ist, sozusagen eines geschäftsführenden Ausschusses des Plenums der Richterschaft eines Gerichts, und nicht etwa Präsidentenwerk oder sonst Werk der Justizverwaltung. Ich hatte dies mit einer weiteren Norm des Grundgesetzes in Verbindung gebracht: "Die rechtsprechende Gewalt ist den Richtern anvertraut« (Art. 92 GG). Die Wahrnehmung der eigenen Angelegenheiten in dieser Form sichere in besonderer Weise die Unabhängigkeit der Richterinnen und Richter, zu der Art. 97 GG sagt: »Die Richter sind unabhängig und nur dem Gesetz unterworfen «.

französischen und italienischen Kollegen können sich immer - zu Recht - darüber aufregen, dass bei ihnen gelegentlich der Gerichtspräsident in Einzelfällen in die Geschäftsverteilung eingreift, insbesondere dem einen Richter viel, dem anderen wenig Arbeit aufdrückt oder auch einzelne Verfahren an sich zieht. Nun gilt auch dort, wie bei uns gemäß Art. 101 Abs. 1 Satz 2 GG, das Prinzip des gesetzlichen Richters (»Niemand darf seinem gesetzlichen Richter entzogen werden.«) Hier in Deutschland sorgen die Regelungen der $\S \S 21$ a ff. GVG dafür, dass dem auch »in kleiner Münze« Rechnung getragen wird: An jedem Gericht werden, nach abstrakt-generellen Kriterien, vor Beginn jeden Jahres die einzelnen Geschäfte auf die Richter bzw. Spruchkörper verteilt, und dabei bleibt es - von wenigen gesetzlich zugelassenen Ausnahmen abgesehen - verlässlich für die Dauer des Geschäftsjahres; eine unzulässige Abweichung bildet bekanntlich einen absoluten Revisionsgrund.

Dies hatte ich den Kollegen aus ganz Europa, die zum Zwecke der Rechtsvergleichung und des Berichts über die nationalen Praktiken zusammengekommen waren, gerade en detail erläutert und hierzu den mitgebrachten Geschäftsverteilungsplan des Landgerichts Lübeck für das laufende Jahr, ein Opus von cirka 50 Seiten, dargestellt und herumgereicht. Die Kolleginnen und Kollegen waren tief beeindruckt. Dies um so mehr, als sie auch noch gehört hatten, dass der Geschäftsverteilungsplan das Werk eines gewähl-
Im Folgenden wurde der ebenfalls anwesende Court Manager eines großen Londoner Gerichts befragt, wer denn dort die Geschäfte auf die Richter verteile. Und er antwortete mit großer Selbstverständlichkeit: »Ich«. Die englischen Richter würden sich, so sagte er - unter Berufung gerade auf ihre Unabhängigkeit - dagegen verwahren, in so banale Verwaltungsdinge wie die

Man kann also, so zeigte sich, die Frage »Wie sichere ich institutionell die Unabhängigkeit der Richter? « und damit auch die dahinter stehende Frage »Wie organisiere ich eine effiziente, von den Bürgerinnen und Bürgern akzeptierte Rechtspflege, die von unabhängigen Richterinnen und Richtern wahrgenommen wird? « in sehr unterschiedlicher Weise beantworten.

Wie auch immer die Antwort ausfällt, sie muss dem Basisgrundrecht einer gemeinsamen europäischen Rechts- und Justizkultur, nämlich Art. 6 der Europäischen Konvention zum Schutze der Menschenrechte und Grundfreiheiten (EMRK) Rechnung tragen:

»Jede Person hat ein Recht darauf, dass über Streitigkeiten in bezug auf ihre zivilrechtlichen Ansprüche und Verpflichtungen oder über eine gegen sie erhobene strafrechtliche Anklage von einem unabhängigen und unparteiischen, auf Gesetz beruhenden Gericht in einem fairen VerVerteilung der Geschäfte einbezogen zu werden. fahren, öffentlich und innerhalb angemessener Frist verhandelt wird.«

Unter betriebswirtschaftlichen und fiskalischen Vorzeichen - die »offizielle« Justizreformdebatte in Deutschland

Auf den ersten Blick könnte man meinen, es ist nicht verwunderlich, dass in Deutschland seit geraumer Zeit eine Debatte über die Reform der Struktur der Justizbehörden geführt wird, entspricht doch die deutsche Justizverwaltungsstruktur grundsätzlich nach wie vor einem vordemokratischen, im Wesentlichen im 19. Jahrhundert geprägten (»napoleonischen «) Modell. Sie ist hierarchisch konzipiert, oberste Verwaltungsinstanz der Gerichte und Behörden der "Dritten Gewalt" ist - im gewaltengeteilten Staatssystem (!) - das Justizministerium, also ein Teil der Exekutive. Die einzelnen Gerichte werden grundsätzlich nach wie vor durch Präsidenten und Direktoren geleitet, die - neben ihrer Richtertätigkeit - ebenfalls Angehörige der Exekutive sind. Auf die Gerichte werde ich mich übrigens im Folgenden konzentrieren, da sie mir aus eigener Anschauung vertrauter sind, die Staatsanwaltschaften werden allenfalls gestreift.

Eine grundlegende, in Richtung demokratieadäquater Strukturen zielende Reform der Organisation und Verwaltung der Justiz war aber in Deutschland lange nicht Gegenstand der Debatten und der tatsächlichen Reformschritte. (Inzwischen findet die substantielle, radikale Debatte statt, ich werde darauf weiter unten im zweiten Teil, eingehen).

Die Reformdiskussion in Deutschland nahm, wie gesagt, eine ganz andere Richtung. Auslösend hierfür waren Ende der Achtziger-, Anfang der Neunzigerjahre des vorigen Jahrhunderts zum einen, stark an Vorgaben aus der Industrie orientiert, Organisations-Reformvorhaben in der allgemeinen Verwaltung; zum anderen waren Auslöser die Klagen von »Justizkunden «, von Ministern und Parlamentariern über veraltete Binnenstrukturen der Justiz und vor allem über zu lange Prozessdauer. Dieses Ursachenbündel und die daraus hergeleitete Stoßrichtung für Ver- 
änderungen klingen viel banaler, sind es aber nicht. Denn auch hier werden unmittelbar kritische Punkte zum Thema »Unabhängigkeit« berührt, wie noch zu zeigen sein wird.

Zunächst sollte man sich aber ansehen, wie Gerichte klassischerweise in der Bundesrepublik Deutschland aufgebaut, organisiert und verwaltet waren (und zum großen Teil auch noch sind). Zunächst ist festzuhalten, dass die grundlegenden Organisations- und, wenn man so will, Verwaltungsstrukturen der Justiz für ihre Kernaufgabe, nämlich das Durchführen von Gerichtsverfahren, von Prozessen, relativ klar und eindeutig im Gerichtsverfassungsgesetz (GVG) und einigen Annex-Gesetzen (z. B. dem Rechtspflegergesetz) geregelt sind. Es gibt Richter, Rechtspfleger, Urkundsbeamte der Geschäftsstelle, Staatsanwälte. Sie haben, jeweils innerhalb eines Hauses und im Verhältnis zueinander, klar definierte $\mathrm{Zu}$ ständigkeiten und Funktionen.

Und das Entscheidende ist: Die Richterinnen und Richter (in sachlicher Hinsicht auch die Rechtspflegerinnen und Rechtspfleger) führen ihre Verfahren in Unabhängigkeit, kein Präsident, kein von diesem mit der Verwaltung des Hauses betrauter Beamter kann ihnen Weisungen erteilen. Man kann diese Selbstverständlichkeit, die zugleich eine große kulturelle Errungenschaft ist, nicht genug betonen, denn daraus ergibt sich, dass ein Gericht fundamental anders arbeitet als eine (hierarchisch-monokratisch aufgebaute) Verwaltungsbehörde. Unter dem Dach eines Gerichts arbeiten so viele einzelne "Gerichte" nebeneinander, wie es der Zahl nach Richterinnen und Richter (Rechtspflegerinnen und Rechtspfleger) oder Spruchkörper gibt. Jeder von ihnen bzw. jeder Spruchkörper ist »das Gericht «.

Das war (und ist, soweit es solche noch gibt) ganz anders am ganz kleinen Amtsgericht, einem Amtsgericht mit nur einem Richter. Dieser führt nicht nur jeden einzelnen Prozeß als Vorsitzender, sondern leitet mit Selbstverständlichkeit auch die Verwaltung des Gerichts. Hier fallen court management (Gerichtsverwaltung) und case management (Prozeßgestaltung) in einer Hand zusammen. Das ist an einem größeren, ausdifferenzierten Gericht anders. Insoweit wären m.E. die Erwägungen von Rosemary Barberet (in diesem Heft) noch zu differenzieren. Ist es schon problematisch, die Gerichtsverwaltung exekutivisch veranlaßt zu gestalten und ggf. zu reformieren, so ist dies vollends ausgeschlossen für die Prozeßgestaltung im Einzelfall. Diese obliegt dem einzelnen Richter/der einzelnen Richterin in ihrer/seiner Unabhängigkeit und ist nur durch den Gesetzgeber vorzugeben Und selbst eine Gesetzesreform, etwa in Richtung einer Straffung und Beschleunigun von Verfahren, wird nur erfolgreich sein(d.h. in der Praxis umgesetzt werden), wenn sie plausibel ist und durch überzeugende einführende Fortbildung begleitet wird, noch besser: wenn das Gesetz die zuvor erprobte und wissenschaftlich ausgewertete »neue« Praxis fortschreibt.

Nun sind heute die ganz kleinen Gerichte eher die Ausnahme, überdies sind sie immer wieder in ihrer Existenz bedroht. Die Mehrzahl der Gerichte (und Staatsanwaltschaften) sind recht große $B e$ hörden. Je größer sie sind und je mehr »arbeitende Einheiten « (bei Gericht etwa definiert durch die Richterin/den Richter und die mit ihr/ihm kooperierenden Rechtspflegerinnen/Rechtspfleger und Urkundsbeamtinnen/Urkundsbeamten der Geschäftsstelle), desto mehr kommt die Neigung und sicher auch das Bedürfnis - nach vereinheitlichenden, auch zeit- und arbeitsökonomischen Strukturen und insbesondere einer einheitlichen Verwaltung auf. Dem entsprechend hat sich seit Inkrafttreten der Reichsjustizgesetze, zu denen auch das GVG gehört, im Jahre 1879 eine Tradition der Gerichtsverwaltung entwickelt, die sicher zunächst und in erster Linie dienende Funktion gegenüber den Rechtsprechungsabteilungen des Gerichts hatte, die aber, wie wir spätestens seit Max Weber für die Verwaltung ganz allgemein wissen, auch ihre Eigendynamik entfaltet.

Der Vollständigkeit halber sei an dieser Stelle kurz eingefügt, wie die Verwaltung eines Gerichts - am Beispiel eines mittelgroßen Landgerichtsbezirks in Schleswig-Holstein - mit circa 110 Richterinnen und Richtern und insgesamt 700 (wenn man die Referendarinnen und Referendare hinzurechnet, circa 1000) Mitarbeiterinnen und Mitarbeitern- aussieht. Ich halte dies deshalb für hilfreich, weil vielfach die Debatte um Organisationsreformen in der Justiz von Personen geführt wird, denen die Anschauung der Ausgangsverhältnisse fehlt, und zwar aus ganz unterschiedlichen Gründen. Parlamentarier und Ministerialbeamte, insbesondere solche aus anderen Verwaltungen als der Justiz, sind es gewohnt, sich Behörden und Verwaltungen jeweils insgesamt als hierarchisch-monokratisch organisierte Einrichtungen vorzustellen; es fällt ihnen schwer, sich auszumalen, dass der gesamte eigentliche Rechtsprechungsbereich, der den Richterinnen und Richtern in Unabhängigkeit anvertraut ist, dem Hineinwirken der Behördenleitung und praktisch jeder Dienstaufsicht entzogen ist. Richterinnen und Richtern selbst fehlt es - bedauerlicherweise - häufig an der Anschauung hinsichtlich der Verwaltung ihrer eigenen Gerichte, weil mit dem traditionell gewachsenen »Wuchern « der Verwaltung sie sich vielfach - insoweit im Ergebnis dem oben geschilderten »britischen Modell« nahe - daran gewöhnt haben, die von Ihnen wahrgenommene Verantwortung für das gemeinsame Ganze des Gerichts auf ihren eigenen richterlichen Kernanteil zu reduzieren. Im Extrem ist das die vielfach karikaturhaft dargestellte Haltung des Richters, der, von den Beratungen und Sitzungen abgesehen, nur sein Dienstzimmer und dort den »Eingangsbock « (für die Akten), seinen Schreibtisch und den »Ausgangsbock« kennt und daher allenfalls den Wachtmeister sieht, der ab und zu - als die Konzentration störendes Element - das Dienstzimmer betritt. Dabei ist aus dem Blick geraten, was in der täglichen Praxis an sich eine Selbstverständlichkeit bedeutet: dass nämlich die Richterin/der Richter in funktionaler Hinsicht »Vorgesetzter « der Kolleginnen und Kollegen sind, mit denen sie in der Geschäftsstelle zusammenarbeiten: Es sind dies Urkundsbeamtinnen und Urkundsbeamten, ähnlich die Schreibkräfte, die die Verfügungen, Beschlüsse und Urteile der Richterin/des Richters schließlich geschrieben haben. Warum war dies vielfach außerhalb des Blicks? Die Schreibkräfte waren, von der sonstigen Geschäftsstellentätigkeit gesondert, in großen Kanzleien, zu Schreibpools zusammengefasst, sie erhielten ihre Aufgaben vom Leiter der Kanzlei zugeteilt, bei dem diese jeweils aus den Geschäftsstellen, zugetragen durch die Wachtmeister, aufliefen. Das Ganze vollzog sich, wie sämtliche anderen Aufgaben des inneren Dienstes der Behörde in der Verantwortlichkeit des geschäftsleitenden Beamten. Er sorgt dafür, dass allen Richterinnen und Richtern die erforderliche Infrastuktur zur Verfügung steht: Dienstzimmer, Verhandlungsräume, Schreib- und Diktiergerät, heute natürlich Informationstechnologie, und vor allem: Mitarbeiterinnen und Mitarbeiter, und dies alles gerecht verteilt bei knappen Ressourcen. Der geschäftsleitende Beamte gehört nach wie vor mit dem für die richterlichen Personalangelegenheiten und die richterbezogenen sonstigen Verwaltungsangelegenheiten der Gerichte des Bezirks zuständigen richterlichen Verwaltungsreferenten (Präsidialrichter, richterlichen Verwaltungsreferenten) und dem (allgemeinen) Verwaltungsreferenten, der für die Belange der Amtsgerichte des Bezirks, für sämtliche nichtrichterlichen Bediensteten und für den Haushalt des Landgerichtsbezirks zuständig ist, dem Präsidenten, und dem Vizepräsidenten, als dessen ständigem Vertreter, zur Leitungsspitze des Landgerichts. Daneben gibt es für einzelne Fachgebiete (Presse- und Öffentlichkeitsarbeit, Referendarausbildung, Bibliothek, Notarangelegenheiten, IT- und Organisationsangelegenheiten, Gleichstellung, Datenschutz o. a.) richterliche Referentinnen und Referenten, die - wie Präsident, Vizepräsident und richterlicher Verwaltungsreferent - die Verwaltungsaufgaben neben ihrem (mehr oder weniger umfangreichen) Richterdezernat ausüben. Das Prinzip hierbei ist, dass Verwaltungsaufgaben aus richterlicher Perspektive wahrgenommen werden, daher die Besetzung der Funktionen mit Richterinnen oder Richtern, daneben auch in starkem Umfang mit Rechtspflegerinnen und Rechtspflegern. Bemerkenswert ist dabei, dass die Funktion des richterlichen Personalreferenten auf Zeit (in der Regel circa drei Jahre) wahrgenommen wird. Die Tätigkeit dieses »Gerichtsmanagers " wird on the job trainiert. Sie bietet große Chancen für die persönliche Entwicklung besonders qualifizierter, schon erfahrener junger Richterinnen und Richter und trägt $\mathrm{zu}$ einer 
ständigen inneren und äußeren Erneuerung der sonst eher auf Kontinuität angelegten Leitung des Gerichts bei.

Dies ist der organisatorische Hintergrund, vor dem die - bescheidenen, aber sehr wirksamen bis heute andauernden Reformvorhaben der Neunzigerjahre des vergangenen Jahrhunderts zu sehen sind.

\section{Konsequenzen aus Unternehmensberatung für die Justiz}

Es begann mit einem Bund-Länder-Projekt, noch hervorgegangen aus dem früheren, von Strempel geleiteten Referat für Rechtstatsachenforschung im Bundesministerium der Justiz (BMJ), mit der sog. Kienbaum-Studie zur Organisation der Amtsgerichte, durchgeführt von der gleichnamigen Unternehmensberatung. Der zutreffenden Analyse der »zerhackten « Arbeitsabläufe mit vielfältigen vermeidbaren Herumtrage- und Liegezeiten der Prozessakten folgte in allen Ländern der Bundesrepublik Deutschland, beschleunigt in den neuen Ländern in der ehemaligen DDR, sozusagen die Rückbesinnung auf das GVG-Modell: Weg von den stark »verwaltungslastigen « Organisationsformen, weg etwa von Großkanzleien, hin $\mathrm{zu}$ - nunmehr Serviceeinheiten genannten - arbeitsfähigen, die Schreibkräfte integrierenden und fortbildenden Geschäftsstellen und vor allem $\mathrm{zu}$ einer intensiven, die Verantwortlichkeit der Richterinnen und Richter, Rechtspflegerinnen und Rechtspfleger fordernden Zusammenarbeit zwischen diesen "Entscheidern « und den Mitarbeiterinnen und Mitarbeitern in der Serviceeinheit; dies in Verbindung mit der Einführung moderner Informationstechnologie, insbesondere vernetzter PCs mit arbeitsplatzbezogener justizspezifischer Software.

Ein weiteres großes Projekt, das inzwischen in zahlreichen Ländern (darunter Schleswig-Holstein) ganz oder teilweise verwirklicht ist, betrifft die Kosten- und Leistungsrechnung (KLR), die es der einzelnen Richterin/dem einzelnen Richter wie dem Gericht und der Justiz insgesamt (erstmals) ermöglicht, die tatsächlichen Kosten einzelner Verfahren zu verfolgen, die Kostenstruktur verschiedener Verfahrensarten zu errechnen und dadurch verlässlichere Daten für die Haushaltsaufstellung und für die Personalbedarfsberechnung an der Hand zu haben. Gleichzeitig kann die KLR zu Kostenbewusstsein bei den Richterinnen und Richtern führen. Für die Einführung regelrechter Budgets, die in Zukunft den einzelnen Gerichten zur Verfügung stehen könnten, ist die KLR eine Voraussetzung.

In vergleichbarer Weise und mit der Kostenund Leistungsrechnung verzahnt, läuft ein weiteres bundesweites Reformvorhaben, die veränderte Personalbedarfsberechnung. Während bisher - in durchaus fragwürdiger und von den Richtern immer wieder kritisierter Weise - eine Art »Stückak- kord « (nämlich Sollzahlen für bestimmte Arten von gerichtlichen Verfahren pro Richter und Jahr) den Personalbedarfsberechnungen zu Grunde gelegt waren, hat nunmehr die ständige von der Justizministerkonferenz (JMK) eingesetzte Pensenkommission - auch hier in Zusammenarbeit mit einer Unternehmensberatung, nämlich $A r$ thur Anderson - ein analytisches, auf Zeiterfassung durch eine Vielzahl von Richterinnen und Richtern in repräsentativ ausgewählten Ländern beruhendes Verfahren entwickelt (Pebby), das nunmehr, nach Feinabstimmung in den Ländern, bundesweit eingeführt werden soll. Das System ermöglicht im Übrigen eine Koordinierung mit der Justizstatistik, die bisher mehr ein großer Datenfriedhof war, als dass sie eine bedarfsgerechte Personalplanung ermöglichte.

Sind dies eher in den Ländern flächendeckend und bundesweit abgestimmt durchgeführte Reformvorhaben, so gibt es weitere Vorhaben, die sich jeweils an den einzelnen Gerichten auswirken. Hier seien - ohne Anspruch auf Vollständigkeit noch die Einführung von Instrumentarien der Personalentwicklung und des Qualitätsmanagements genannt, ferner - soweit nicht ohnehin vorhanden - die (verstärkte) Einführung von AbteilungsStrukturen, um die Leitungsspannen kleiner und handhabbar zu halten; und schließlich die Einführung anderer Formen der Gerichtsleitung (court management). Die Diskussion hier beruht wesentlich auf den Erhebungen und Mitteilungen von Röhl aus den USA. Zum court management gibt es meines Wissens real existierende Versuche lediglich in Hamburg am Amtsgericht und am Oberlandesgericht. Da dort jeweils Richter in die neu (und zusätzlich) geschaffenen Ämter des Court Managers berufen wurden und diese auch mit hoher Wahrscheinlichkeit nicht auf Dauer in diesen Funktionen verbleiben dürften, hielte ich es eher für angebracht - etwas bescheidener - hier von einer Fortführung des oben beschriebenen, m. E. äußerst erfolgreichen Modells des auf Zeit berufenen richterlichen Verwaltungsreferenten $\mathrm{zu}$ sprechen, allerdings mit der positiven Begleiterscheinung, dass für die anspruchsvolle und zeitaufwendige Leitungsaufgabe eine leistungsbezogene Zulage in der Form gezahlt wird, dass die Amtsinhaber in gehobene richterliche Besoldungsgruppen ( $\mathrm{R} 2$ statt $\mathrm{R} 1$ beim Amtsgericht, $\mathrm{R}$ 3 statt R 2 beim OLG) eingruppiert sind.

\section{Impulse für eine Grundsatzdebatte}

Wie ich im Vorstehenden absichtsvoll ohne jede Kommentierung beschrieben habe, sind die Anstöße für die genannten Reformvorhaben nicht aus den Gerichten selbst, schon gar nicht aus der Richterschaft selbst, gekommen. Vielmehr beruhten sie auf Anstößen durch die Landesjustizverwaltungen, gestützt durch wissenschaftliche Untersuchungen mehr organisationssoziologischen und betriebswirtschaftlichen Charakters. Es wird nicht verwundern, dass auch die Finanzminister und der jeweilige Haushaltsgesetzgeber all dies aktiv begleitet, wenn nicht mit angestoßen haben. Das Bild rundet sich, wenn wahrheitsgemäß und summierend hinzugefügt wird, dass - in Schleswig-Holstein kann ich dies aus eigener Anschauung berichten - die Überprüfungs- und Re-
(1)

(1)

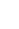


der Richterschaft selbst geführt. Im Prozess der Reform hat sich das entwickelt, was - nach der reinen Lehre - Voraussetzung für dessen Beginn gewesen wäre. Hieran haben die richterlichen Berufsorganisationen (Richterbund, Richter/-innen in ver.di, Neue Richtervereinigung) und die Richtervertretungen großen Anteil. Aber der Grat der »Richterverträglichkeit« der eingeführten neuen Instrumentarien ist wohl durchaus von Land $\mathrm{zu}$ Land der Bundesrepublik sehr unterschiedlich.

\section{Es fehlt noch ganz anderes: die substantielle Justizreform}

In anderen Staaten mit vergleichbarer Justizstruktur (und vergleichbaren Problemen) hat man die Reformvorhaben ganz anders, nämlich im Zusammenhang mit einer Reform an Haupt und Gliedern der Justiz angefasst. Ich nenne beispielhaft die Niederlande. Dort ist im Verlaufe der Neunzigerjahre des vergangenen Jahrhunderts die gesamte Verwaltung der Gerichte in Richtung einer Selbstverwaltungsstruktur entwickelt worden, innerhalb derer die weiteren Reformziele-, -prozesse und -schritte durch Gremien der Richterschaft selbst entwickelt wurden.

Dies steht in einem bestimmten europäischen historischen Kontext: Ausgehend von dem (bescheideneren) französischen und dem (umfassenden) italienischen Modell aus der Zeit nach 1945 haben sich in anderen (post-faschistischen) Staaten, nämlich Spanien und Portugal modellhaft Strukturen der Justizverwaltung entwickelt, die demokratie-adäquat sind. Nach 1989 sind die früher kommunistischen Staaten in Mittel- und Westeuropa mit vielfach interessanten Konstruktionen (die allerdings durchaus mit Vorsicht aus der Nähe zu betrachten sind) gefolgt. In Westeuropa sind neuerdings die Niederlande (s. o.) und Dänemark zu nennen. Auch Großbritannien ist auf einem interessanten Weg.

Unterstützt wird dies durch Vorgaben des Europarats. Hier ist insbesondere die europäische Charta für ein Richterstatut von 1998 zu nennen, die allerdings nur den Stellenwert eines Expertenpapiers hat (Ich habe an der Erarbeitung teilgenommen), mit ganz anderem Gewicht die Empfehlung des Ministerkomitees Nr. (94) 12 vom 13.10.1994 und die Entschließung des Ministerkomitees vom 18.12.2002 (Entschließung Res (2002) 12) zur Einsetzung der Europäischen Kommission für die Wirksamkeit der Justiz (CE$\mathrm{PEJ}), \mathrm{zu}$ der es jetzt auch noch eine begleitende Richter-Kommission gibt.

Das Modell lässt sich dadurch kennzeichnen, dass die Gesamtverwaltung der Gerichtsangelegenheiten nicht mehr durch Justizministerien geschieht, sondern durch »Oberste Richterräte « als Selbstverwaltungsorgane der Richterschaft, freilich unter Einschluss hinzugewählter Repräsentanten der gesellschaftlichen Pluralität (im Ein- zelnen unterschiedlich: Parlamentarier, Hochschullehrer, Angehörige anderer Justizberufe wie Rechtsanwälte und Notare).

Hier fällt mittlerweile die Bundesrepublik Deutschland stark aus dem Rahmen. Bösartige Kritiker dieses Zustandes haben es auf die polemische Frage zugespitzt, ob wohl die Bundesrepublik Deutschland mit ihren Justizverwaltungsstrukturen den Anforderungen an eine demokratie-adäquate Verwaltungsorganisation der Justiz genügen würde, die, in Anlehnung an das genannte Expertenpapier des Europarates, den zukünftigen EU-Beitrittsländern abgefordert werden.

Ehe ich darauf eingehe, in welche Richtung eine solche, substantielle Justizreform erfolgen könnte, möchte ich aber doch noch ein gutes Wort für die Gesamtorganisation der Justiz in der Bundesrepublik Deutschland, insbesondere deren Verwaltung, einlegen, weil nämlich inzwischen zu der fragwürdigen vorkonstitutionellen Grundstruktur einige vorbildliche Elemente hinzugetreten sind.

- Ich hatte bereits oben darauf hingewiesen, dass die Präsidien der Gerichte als Selbstverwaltungsorgan bereits jetzt die wichtigste Leitungsaufgabe eines Gerichts erfüllen, nämlich die $\mathrm{Zu}$ sammensetzung der Spruchkörper und die richterliche Geschäftsverteilung. Die Präsidien haben seit 2000 überdies ein verbessertes Wahlrecht, es ist nunmehr auch geklärt, dass sie richteröffentlich tagen dürfen.

- Als Vorstufe richterlicher Selbstverwaltung gibt es, freilich in den Ländern unterschiedlich ausgestaltet nach Intensität und Zuständigkeitsfeldern, Formen der Mitbestimmung nach dem Personalvertretungsgesetz. Die richterliche Mitbestimmung wird durch die Richter- und Präsidialräte ausgeübt.

- Ein Zuständigkeitsfeld für die in anderen Staaten existierenden Obersten Richterräte sind die Disziplinarangelegenheiten der Richter. Hierfür sind dort besondere Kommissionen gebildet. In der Bundesrepublik Deutschland sind die gewichtigeren Disziplinarangelegenheiten in der Weise in die Hände von Richtern gelegt, dass hierüber Richterdienstgerichte entscheiden, also nicht - wie anderswo durchaus üblich Verwaltungsorgane. Überdies besteht für die einzelnen Richterinnen/den einzelnen Richter die Möglichkeit, nach $\S 26$ Abs. 3 des Deutschen Richtergesetzes (DRiG) von sich aus das Richterdienstgericht anzurufen

- Die Berufung der Richterinnen und Richter in ihr Amt erfolgt für die Bundesrichter und in zahlreichen Ländern der Bundesrepublik Deutschland für die Richter, die im Landesdienst stehen, nicht durch den zuständigen Minister (in der Regel: den Justizminister) allein, sondern im Zusammenwirken mit parlamentarischen Richterwahlausschüssen, in denen zum Teil auch Richter mitwirken; in jedem Falle nehmen die Präsidialräte als Organ der Mitbestimmung vor Auswahl und Ernennung Stellung.
Es sei noch - durchaus nicht am Rande - erwähnt: Mit der Gerichtsverfassungs- und Organisationsstruktur des Bundesverfassungsgerichts (BVerfG) existiert geradezu modellhaft eine Struktur für ein demokratisch konzipiertes, selbstverwaltetes Gericht.

Das alles ändert aber m. E. nichts daran, dass eine grundlegende Reform der Justizverwaltung auch in Deutschland unabweisbar ist.

\section{Die Teile zu einem Ganzen}

1. Es geht dabei um die Reform

- der internen Verwaltungsstrukturen der Gerichte

- der Gerichte (und Staatsanwaltschaften) der Länder und des Bundes insgesamt

- des Verhältnisses Justiz/Justizministerien/Parlamente.

\section{Im Einzelnen:}

2. a) Vielfach wird bei der Diskussion und Konzeption demokratie-adäquater Verwaltungsstrukturen der Justiz der Fehler gemacht, gleich auf nationaler oder (im föderalen Staat) auf Länderebene zu beginnen.

Es empfiehlt sich aber, »vor Ort«, d. h. bei den einzelnen Gerichten zu beginnen, zumal schon heute dort mit den Präsidien eine ausbaufähige Struktur vorliegt.

Also: Die Zuständigkeiten der Präsidien der Gerichte sind in Richtung einer umfassenden (Selbst-)Verwaltungskompetenz auszubauen, die Präsidentin/der Präsident wird in Zukunft (wie beim BVerfG) in allen Verwaltungsangelegenheiten Geschäftsführer des Präsidiums sein.

b) In diesem Sinne des »basisnahen « Beginns des Aufbaus einer demokratie-adäquaten Form der Justizverwaltung wäre zu bedenken, ob nicht am Beispiel der Ordentlichen Gerichtsbarkeit etwa die Präsidien der Landgerichte und der Oberlandesgerichte die genannten (Selbst-)Verwaltungsfunktionen von den im jeweiligen Bezirk befindlichen Gerichten, im Sinne einer Verbund-(Selbst)verwaltung übertragen erhalten.

3. a) Die Justiz ist in Deutschland im Wesentlichen als Länderangelegenheit verfasst und organisiert.

Es sind daher, neben einem entsprechendem Gremium für die Bundesgerichte (?), Landesgerichtsbarkeitsräte (unter Berücksichtigung der Besonderheiten entsprechend für die Staatsanwaltschaften, oder gemeinsam?) einzurichten.

Hier wird sich die Frage stellen, ob sie für jede Gerichtsbarkeit einzeln zu errichten sind oder für alle Gerichtsbarkeiten zusammen (wohl vorzuziehen; dann wird freilich für eine hinreichende Re- 
präsentanz der besonderen Gerichtsbarkeiten Sorge zu tragen sein).

Die Landesgerichtsbarkeitsräte sind mit einer qualifizierten Mehrheit (am Besten wohl 2/3) von Richtern, im Übrigen mit vom Parlament gewählten Vertretern mit näher zu bestimmender Qualifikation zu besetzen.

M. E. völlig ungeklärt, aber dringend zu lösen ist das Problem, wie die Landesgerichtsbarkeitsräte, die in den einzelnen Ländern und für die Bundesgerichte bestehen, insgesamt auf Bundesebene koordiniert werden. Dies ist ein Problem, das sich speziell im föderalen Staat stellt. Soweit mir bekannt, sind die Modelle in anderen Ländern im Wesentlichen auf zentrale Strukturen zugeschnitten. Aber hier wird man, angefangen von »ZDF- oder ARD-Modellen « bis zu den Strukturen der Programmkommission der Deutschen Richterakademie, sicher ausbaufähige Modelle finden.

b) Entscheidend für die richterlich zu wählenden Mitglieder der Landesgerichtsbarkeitsräte sind - das Wahlsystem,

- die Wahlkollegien und

- die Frage "geborener Mitglieder«.

Das Wahlsystem kann nur das (proportionsgerechte, minderheitenfreundliche) Verhältniswahlsystem sein.

Für Gleichheit im Zähl- und Erfolgswert der Richterstimmen ist es wichtig, dass möglichst jede Richterin/jeder Richter mit gleicher Stimme an der Wahl aller zu wählenden Mitglieder beteiligt ist. Es darf nicht sein, dass etwa die (wenigen) Richterinnen und Richter an den Oberlandesgerichten ebenso viele Vertreterinnen/Vertreter entsenden wie die (wesentlich größere Zahl der) Amtsrichterinnen und Amtsrichter. Es ist also ein einziges (einheitliches) Wahlkollegium zu bilden.

Geborene Mitglieder müssen die Ausnahme sein. Sie können sonst die Stimmverhältnisse unverhältnismäßig beeinflussen.

c) Auf die Gerichtsbarkeitsräte gehen alle laufenden Geschäfte der Justizverwaltung über, das sind insbesondere

- Personalangelegenheiten (einschließlich Dienstaufsicht und - ggf. vorbereitend - Disziplinarangelegenheiten)

- Aus- und Fortbildung (nur der Richter?), einschließlich Prüfungswesen (?)

- Haushaltsangelegenheiten

4. Die Kompetenzen der parlamentarischen Richterwahlausschüsse bleiben unberührt / sind dort einzuführen / zu erweitern, wo solche noch nicht oder unzureichend vorhanden sind.

Im Einzelnen stellen sich unter anderem folgende Fragen/Probleme: a) Sollen die Richterwahlausschüsse weiter gemeinsam mit der zuständigen Ministerin/dem zuständigen Minister oder in Zukunft gemeinsam mit dem Gerichtsbarkeitsrat entscheiden? Im letzten Fall, müsste insoweit das Grundgesetz geändert werden.

b) Im ersten Fall: Wo soll die Geschäftsführung für die Richterwahlausschüsse liegen? (Beim Parlament, weiter bei der Ministerin/dem Minister, bei den Gerichtsbarkeitsräten?)

c) Soll die parlamentarische Richterwahl nur für die (Eingangs-)Wahl bei Eintritt in den Beruf oder auch in Funktionsstellen stattfinden? (Sonst wäre für letztere die Alternative: Wahl vor Ort durch die Selbstverwaltungsgremien.)

5. Dem Justizministerium werden weiter Aufgaben verbleiben:

- Justitiariat der Regierung

- Gesetzgebungsangelegenheiten

- Koordinierung der Gesetzgebung und verbleibender Justizministerialangelegenheiten im Verhältnis Bund/Länder

- Justizvollzug

- Gnadenwesen

- Notardienstaufsicht

- internationaler Rechtsverkehr und (nunmehr koordiniert mit dem bundesweiten Koordinationsgremium der Gerichtsbarkeitsräte)

- internationale Angelegenheiten der Rechtspolitik/internationale Juristenfortbildung

6. Von großer Bedeutung wird die (behördenmäßige) Organisation der Gerichtsbarkeitsräte sein.

Selbstverständlich brauchen sie einen Verwaltungs-Unterbau.

Wie bei allen Institutionen, bei denen es eine (ehrenamtliche und/oder auf Zeit bestellte) Vorstandschaft und eine (dauerhafte) faktische Geschäftsführung gibt, wird sorgfältig auf das (Macht-)Verhältnis zwischen gewählten Leitungsorganen und der (dauerhaften? jeweils - teilweise - wechselnden?) Verwaltung zu achten sein.

7. Genau zu überlegen wird sein, ob die Reform "auf einen Schlag « oder in behutsamen Teilschritten durchgeführt werden soll. Für das Erste spricht, dass andernfalls von vornherein eine Verwässerung eintreten könnte; eine radikale Reform braucht nun einmal einen klaren Schnitt (natürlich bei vorzusehenden Übergangsvorschriften). Für das Zweite könnte der Umstand sprechen, dass die quantitativ sehr große, überdies föderal gegliederte Justiz der Bundesrepublik Deutschland einen einmaligen »Schnitt« eventuell nicht verkraftet.

8. Zum Thema »Übergangsstatut « gibt es inzwischen einen ganz anderen, intelligenten Vorschlag aus der Feder des Richters am Bundesverwaltungsgericht Dr. Bernd Brunn. Der Vorschlag, den dieser, wie üblich ausdrücklich als »aus- schließlich die persönliche Meinung des Autors« gekennzeichnet, kürzlich in der Zeitschrift betrifft: justiz (bj) Nr. 76 (= Heft 4/2003), S. 199, mit einer Einführung "Der Zustand des Gerichtsverfassungsrechts« (S. 196 ff.) veröffentlicht hat, ist nicht nur wegen seines Inhalts interessant, sondern auch wegen der Person des Autors. Brunn ist derzeit abgeordnet zum BMJ und dort mit der Sichtung fortgeltenden Bundesrechts befasst. Der Umstand, dass er zum Thema "Gerichtsverfassung « auf einen äußerst geringen und vielfach nicht gerade "grundgesetz-kompatiblen " Normenbestand gestoßen ist, gibt ihm Anlass, den Entwurf eines neuen GVG vorzulegen, das in seinen (grundlegenden) ersten Paragraphen Teile der hier skizzierten Reformüberlegungen aufnimmt und im Übrigen den Ländern Spielräume eröffnet, neue Formen der »Ordnung des Gerichtswesens« einschließlich der »Beteiligungsrechte der Richter« sowie der »Zuständigkeit für die Aufsicht über Gerichte und Richter« zu erproben.

\section{Weiterführende Hinweise zum Thema}

Die Literatur $\mathrm{zu}$ beiden von mir dargestellten Aspekten des Themas ist Legion.

Da die Diskussion zum zweiten Teil, insbesondere aus den umliegenden Staaten Europas beeinflusst, vor allem in den Berufsorganisationen der Richterinnen und Richter (und Staatsanwältinnen und Staatsanwälte) geführt und befördert worden ist, nenne ich zur vertiefenden Lektüre (in der Reihenfolge der Aktualität wie auch meiner Identifikation und Sympathie);

- Richterinnen und Richter, Staatsanwältinnen und Staatsanwälte in der Vereinten Dienstleistungsgewerkschschaft (ver.di), Sicherung der Unabhängigkeit der Richterinnen und Richter durch verstärkte Selbstverwaltung der Gerichte und der Justiz, verdikt 2/03 (Dezember 2003), S. 19

- Neue Richtervereinigung (NRV), Strukturen einer unabhängigen und demokratischen Justiz, betrifft: justiz Nr. 74 (= Heft 2/2003, S. 60)

- Deutscher Richterbund (DRiB), Diskussionspapier »Selbstverwaltung «, DRiZ 2002, S.

Aus der verfassungsrechtlichen Literatur:

- Thomas Groß, Verfassungsrechtliche Möglichkeiten und Begrenzungen für eine Selbstverwaltung der Justiz, Zeitschrift für Rechtspolitik (ZRP) 1999, S. 361.

- Hans-Ernst Böttcher, Justiz in der Bundesrepublik Deutschland - Richterbild, Justizstruktur, Erforderlichkeit von Reformen, in: Grundgesetz, Mitarbeiterkommentar und Handbuch, hrsg. von Dieter C. Umbach und Thomas Clemens, Heidelberg 2002, Vorbemerkungen vor Art. 92 ff. GG

Der Verfasser ist Präsident des Landgerichts Lübeck 